\title{
Den Begriff mit Leben füllen
}

\section{Der Begriff Nachhaltigkeit ist nur wenigen Managern und Mitarbeitern bekannt - richtig kommuniziert stößt er jedoch auf großes Interesse. Nachhaltigkeits- berichte die dies berücksichtigen, können damit auch zur Mitarbeiterbindung beitragen. Das ist ein Ergebnis einer Befragung, die an zwei Standorten des Axel Springer Verlags durchgeführt wurde.}

$\mathrm{U}$ Von Jutta Hoppe und Niels Ferdinand ber die Rolle der Wirtschaft im Streben nach einer nachhaltigen Entwicklung wird seit dem Gipfel der Vereinten Nationen in Johannesburg wieder viel diskutiert. Von Unternehmen wird dabei gefordert, sich an dem Leitbild des nachhaltigen Wirtschaftens auszurichten, also ihren ökonomischen Erfolg mit ökologischer und sozialer Verträglichkeit in Einklang zu bringen. Immer mehr Firmen schließen sich diesem Leitbild an. Sie erhoffen sich davon vor allem ImagePluspunkte sowie eine Verbesserung ihrer Beziehungen zu so genannten Stakeholdergruppen, wie beispielsweise zu Verbänden, Investoren und Mitarbeitern.

Eine Möglichkeit sowohl unternehmensinterne als auch -externe Interessierte über ihre Bemühungen um eine nachhaltige Entwicklung zu informieren, besteht für Unternehmen in der Veröffentlichung eines Nachhaltigkeitsberichtes oder Sustainability Reports. Ein solcher Bericht wird jedoch nur dann einen Beitrag zur Verbesserung des Unternehmensimages leisten können, wenn die in ihm veröffentlichten Informationen von den Adressaten wahrgenommen und als relevant und glaubwürdig betrachtet werden. Hierzu ist es notwendig, Nachhaltigkeitsberichte auf die Bedürfnisse ihrer Zielgruppen auszurichten. Als wesentliche Zielgruppe von Nachhaltigkeitsberichten bezeichnen viele Unternehmen ihre Mitarbeiter (1). Im Rahmen unserer Diplomarbeit an der Universität Lüneburg haben wir daher untersucht, wie die Kommunikation über das Thema Nachhaltigkeit im Unternehmen verbessert werden kann.

\section{- Breite empirische Studie}

Eine empirische Erhebung beim Axel Springer Verlag, der im Juli 2001 als eines der ersten deutschen Unternehmen einen Nachhaltigkeitsbericht veröffentlichte, stand im Mittelpunkt der Arbeit. Ziel war es, den Informationsbedarf der Mitarbeiter zur Bewertung der Nachhaltigkeit ihres Arbeit- gebers sowie ihre Wahrnehmung des veröffentlichten Nachhaltigkeitsberichtes zu ermitteln. Weiterer Gegenstand der Untersuchung war, welche Informationen aus Sicht der Manager zu vermit-
Offset-Druckerei Ahrensburg des Axel Springer Verlages statt. Die Interviews hatten eine durchschnittliche Dauer von ca. 60 Minuten.

\section{- Nachhaltigkeitsprinzipien sind für Mitarbeiter interessant}

Die Befragung ergab, dass trotz des erst kürzlich veröffentlichten Sustainability Reports der Begriff Nachhaltigkeit innerhalb des Axel Springer Verlages bisher wenigen Interviewpartnern bekannt ist (vgl. Abbildung 1). Viele bezeichneten ihn als verwirrend und wenig greifbar. Ein Mitarbeiter brachte das Problem auf den Punkt: ,Ich finde den Begriff nicht glïcklich. Man hört ihn immer, aber keiner weiß, worum es dabei geht."

Die Befragung zeigte aber gleichzeitig, dass Mitar-

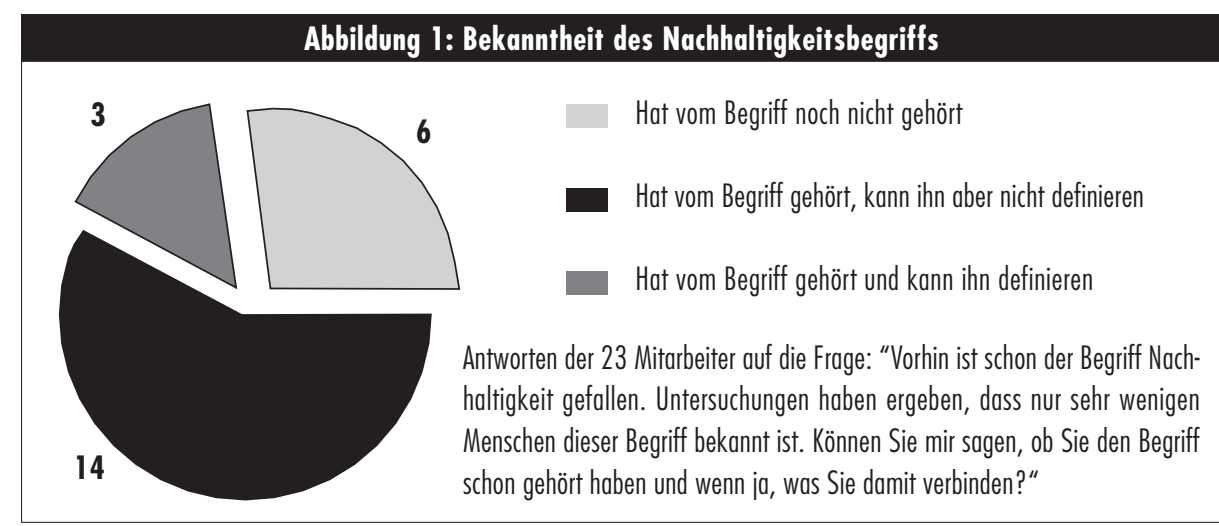

teln sind und wie sie selbst die Wirkung des Berichtes beurteilen. Nachhaltigkeit wird sehr unterschiedlich ausgelegt und definiert. Aus diesem Grund wurde auch der Frage nachgegangen, wie Nachhaltigkeit trotz dieser Ungewissheit zielgruppengerecht kommuniziert werden kann. Dazu wurde untersucht, welche Bedeutung die Kommunikationspartner den unter dem Nachhaltigkeitsbegriff diskutierten Themen zumessen und wie sich deren Sichtweisen unterscheiden.

Die empirische Untersuchung wurde mittels Dialogischer Interviews durchgeführt, das heißt mit Interviewleitfäden ohne Antwortvorgaben. Diese Vorgehensweise gestattet detaillierte Beschreibungen von individuellen Einstellungen und Meinungen und bietet den Befragten so die Möglichkeit, komplexe Zusammenhänge darzustellen. Es wurden 23 Mitarbeiter unterschiedlicher Hierarchiestufen und Tätigkeitsbereiche befragt. Des Weiteren wurden sechs Manager interviewt, die an der Erstellung des Nachhaltigkeitsberichtes beteiligt waren, denen eine Schnittstellenfunktion für die interne oder externe Kommunikation zukommt bzw. die der obersten Leitungsebene angehören. Die Befragung fand von August bis Oktober 2001 im Verlagshaus Hamburg und in der
Quelle: eigene Darstellung

beiter Prinzipien des nachhaltigen Wirtschaftens eine große Bedeutung zumessen. Denn der überwiegenden Mehrheit ist bei der Bewertung eines Arbeitgebers nicht nur das Gehalt wichtig. Vielmehr verlangen sie von einem Unternehmen, sich aktiv um Umweltschutz zu bemühen. Auch soziales Engagement - beispielsweise die Förderung der Chancengleichheit und der Solidarität im Betrieb - ist ihnen äußerst wichtig. Auf der ökonomischen Seite erwarten die befragten Mitarbeiter von ihrem Arbeitgeber, stärker auf den langfristigen Erhalt des Unternehmens als kurzfristig auf höchstmögliche Gewinne abzuzielen.

Betriebe, die ihr Handeln an dem Leitbild Nachhaltigkeit ausrichten und Mitarbeiter gezielt darüber informieren, können nach den Ergebnissen der Befragung mit breiter Zustimmung für ihr Engagement rechnen. Wenn Mitarbeiter erkennen, dass ihr Arbeitgeber nicht nur wirtschaftlich erfolgreich ist, sondern auch ökologischen und sozialen Anforderungen gerecht wird, werden sie leichter zu motivieren sein und sich eher mit ihm identifizieren. 


\section{- Unternehmenseigene Interpretation entwickeln!}

Dies kann jedoch nur gelingen, wenn der Begriff Nachhaltigkeit auf Unternehmensebene mit Leben gefiillt wird. Denn das Thema ist zu abstrakt, um ohne weitere Erläuterungen als Ziel eines Unternehmens vermittelt zu werden. Wollen Betriebe glaubwürdig und effektiv hierüber kommunizieren, sollten sie zunächst definieren, was sie unter dem Begriff verstehen, welchen Beitrag sie zu einer nachhaltigen Entwicklung der Gesellschaft leisten wollen und wie sich das Thema in ihren Zielen und Strategien wiederfinden soll. Dabei ist jedoch zu berïcksichtigen, dass sich Nachhaltigkeit aufgrund der Komplexität der Teilbereiche, deren vielfältigen Wechselbeziehungen und der Ungewissheit künftiger Entwicklungen einer einfachen und abschließenden Zielbestimmung entzieht (2). Das Konzept Nachhaltigkeit stellt daher keine klar definierte Zielvorgabe dar; vielmehr ist es notwendig, eine unternehmenseigene Interpretation von Nachhaltigkeit zu entwickeln und immer wieder an neue Erkenntnisse und Entwicklungen anzupassen. Eine solche unternehmenseigene Interpretation ist von großer Bedeutung, da Mitarbeiter ansonsten zwar Einzelaktivitäten wahrnehmen, diese jedoch nicht als Teil einer Gesamtstrategie erkennen.

Die große Zustimmung zu den Prinzipien des Leitbildes darf dabei nicht darüber hinweg täuschen, dass sehr unterschiedliche Ansichten darüber bestehen, wie diese im Betrieb umgesetzt werden sollten. Um glaubwürdig zu sein, dürfen die hieraus resultierenden Konflikte in einem Nachhaltigkeitsbericht nicht vernachlässigt werden. Er sollte darstellen, wie unterschiedliche Erwartungen im unternehmerischen Handeln berücksichtigt werden und wie mit Konflikten umgegangen wird.

Mitarbeiter werden nur dann zur Umsetzung einer gemeinsamen Nachhaltigkeitsvision motiviert sein, wenn sie ihre eigenen Ansichten in deren Definition einbringen können. Um die Chancen eines nachhaltigen Wirtschaftens nutzen zu können, sollte im Betrieb aus diesem Grund frühzeitig ein Dialog initiiert werden, der Führungskräften und Mitarbeitern einen direkten Austausch über ihre Ansichten und Ideen ermöglicht. Hierzu bietet sich beispielsweise die Bildung eines betrieblichen Nachhaltigkeitsrates als gemeinsames Diskussionsforum an, dem Vertreter unterschiedlicher Tätigkeitsbereiche und Hierarchieebenen angehören sollten. Dieses Forum könnte von Dialogischen Interviews flankiert werden. Nach den Ergebnissen der hier vorgestellten Untersuchung sind sie ein geeignetes Instrument, um die Sichtweisen einer breiteren Gruppe von Mitarbeitern zu erheben und damit deren Berücksichtigung zu ermöglichen. Die Integration der so festgestellten Sichtweisen in den Nachhaltigkeitsbericht kann dadurch unterstiutzt werden, dass auch bei seiner Erstellung Mitarbeiter und Mitarbeitervertreter einbezogen werden. Die namentliche Nennung und/oder Fotos der Beteiligten machen den Bericht für die Kollegen interessanter. Die Mitarbeit bei der Erstellung des Nachhaltigkeitsberichtes kann zudem dazu beitragen, dass die Informationen einen stärkeren Handlungsbezug aufweisen, Handlungsalternativen ersichtlich werden und der Bericht an Glaubwürdigkeit gewinnt.

Eine Steigerung der Glaubwürdigkeit des Nachhaltigkeitsberichtes durch die Einbindung von Mitarbeitern, Angehörigen des Betriebsrates und Vertretern von Nichtregierungsorganisationen wird insbesondere dann gelingen, wenn nicht nur positive Stimmen veröffentlicht werden. Vielmehr sollte das Engagement und das Fachwissen solcher Gruppen für eine kritische Auseinandersetzung genutzt werden. Durch die Einbeziehung unterschiedlicher Meinungen und Sichtweisen kann ein Nachhaltigkeitsbericht der Komplexität des Konzepts Nachhaltigkeit besser gerecht werden und eine differenzierte Auseinandersetzung mit dem Thema demonstrieren.

\section{Anmerkungen}

(1) Insbesondere zu Zielgruppen der Umweltberichterstattung vgl. IÖW / IMUG (Hrsg.): Nachhaltigkeitsberichterstattung. Die Praxis glaubwürdiger Kommunikation für zukunftsfähige Unternehmen. Berlin 2002, S. 58.

(2) Vgl. Enquete-Kommission "Schutz des Menschen und der Umwelt": Konzept Nachhaltigkeit. Vom Leitbild zur Umsetzung, Bonn 1998, S. 28.

\section{Die Autorlnnen}

Jutta Hoppe ist Betriebswirtin und Umweltwissenschaftlerin und arbeitet derzeit an ihrer Promotion zu dem Thema "Stakeholderorientierte Berichterstattung in einer globalisierten Welt". Niels Ferdinand ist Umweltwissenschaftler und freier Berater von Projekten der technischen Zusammenarbeit. Er arbeitet an seiner Promotion zum Umgang mit interkulturellen Unterschieden im Rahmen von Stakeholderdialogen. Kontakt: Arbeitsgruppe Demokratieforschung der Universität der Bundeswehr Hamburg, Holstenhofweg 85, 22043 Hamburg. Tel. 040-6541-2785,

Fax -2009, E-Mail: Jutta.Hoppe@unibw-hamburg.de, niels.ferdinand@unibw-hamburg.de

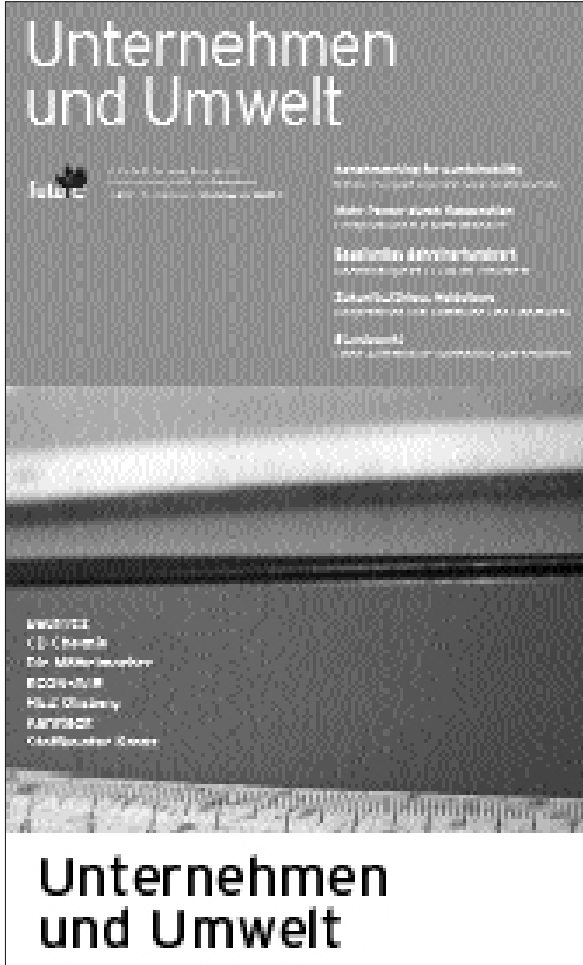

... ist dio Zoitschrift far umwaltorientiorto Unternahmenspolitik von future e.V.

... bie tot Fachinformationen rum Um woltschuts und Best-Practice-Baispide aus Untornohmon.

... biotot viem al im Jahr

Untornahmentakpieb, Litentur- und Vararstaltungthinwoiso.

Mit oinem Schwarpunkttham a in jodam Hoft. Zum Boispiol:

3-4/02: Benchmserking for Sustainebiwity 2/02: Okonnskoting

1/02: Nachhaltigkrit und Gomarbabau

4/01: Nachhaltigkwit im Handwork

3/01: KNimeschutz

2/07: Nachhaltiges Invostmont

1/01: Grone Bauwirtschaft

4/00: Kooporativo Toutilwitschaft

$3 / 00$ : Intogrionto Produktpolitik

2/00: Sort Fact im Umwoitschuts

Bestellen Sie ihr kostenloses Probe-Abo im Internet unter

$\boldsymbol{w} \boldsymbol{w}$. future-er.de

future o.V. Buro Bochum

Am Varanholt 123

44797 Bochum

Fon: 0234/9799513

Fax: $0234 / 9799514$

bochumefuture-øde

www.future-ar.de

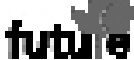


(c) 20I0 Authors; licensee IÖW and oekom verlag. This is an article distributed under the terms of the Creative Commons Attribution Non-Commercial No Derivates License (http://creativecommons.org/licenses/by-nc-nd/3.o/), which permits unrestricted use, distribution, and reproduction in any medium, provided the original work is properly cited. 